\title{
Optimization of Multi-Energy Systems Using the Profile Steering Coordination Framework
}

\author{
Gerwin Hoogsteen, Marco E. T. Gerards, Johann L. Hurink \\ dept. of Electrical Engineering, Mathematics and Computer Science \\ University of Twente \\ Enschede, the Netherlands \\ \{g.hoogsteen, m.e.t.gerards, j.l.hurink\}@utwente.nl
}

\begin{abstract}
Scalable planning and control of individual devices within multi-energy systems is important to support the energy transition. However, multi-energy systems are complex due to relations between different energy carriers on different levels. This paper extends the Profile Steering algorithm with support for such multi-energy systems using distributed optimization, in which individual components can be added. As concrete application, a method to perform load shedding and curtailment, to balance a local district heating network, is presented. Our evaluation shows that a multi-energy system, consisting of a buffer, a CHP, and a heat pump can be optimized within reasonable time and leads to a reduction in export of $51 \%$.

Index Terms-demand side management, multi-energy systems, optimization, smart grids
\end{abstract}

\section{INTRODUCTION}

Many countries are currently developing roadmaps to create a fully sustainable energy supply chain. Next to increasing the share of renewable energy sources (RES), this also requires energy storage. Hereby, seasonal storage is required to supply heat during the colder months. For this often large heat storage vessels are used. The combination of electricity and heat results in a so-called multi-energy system (MES) [1]. Exploiting synergies between different energy carriers results in benefits for the energy system as a whole. As an example, [2] and [3] found significant economic savings when optimal control is used within a MES. These solutions use generic, centralized, solvers to optimally schedule the operation of devices within a MES. The current trend suggests that more complex and intertwined MES will emerge. However, with increasing number of converters, a scalable approach to control such a MES is required as centralized optimization approaches do not scale well. One solution is a double-sided auction to control two energy carriers [4].

In previous work [5], we investigated the use of Profile Steering (PS) [6] to control a MES. In contrast to [4], PS uses model predictive control in a rolling horizon approach. This way, the use of solar PV, small scale battery storage and a combined heat and power (CHP) system, resulted in very little import of electric energy from the main grid. However, the proposed system still exported significant amounts of electricity, which may be resolved by including heat pumps (HPs). The Profile Steering (PS) optimization algorithm as used in [5] does not support this as it can only steer towards energy balance, but not guarantee it.
This paper presents an extension to the scalable Profile Steering (PS) algorithm [6] that allows the integration of multiple converters in a MES. This is done on basis of the coordination algorithm only, in which we focus on efficiently finding a feasible planning rather than overall system efficiency. First we briefly explain the original algorithm and related extensions in Section II. Subsequently, a new scheduling stage is added to the algorithm in Section III to ensure that a feasible solution is found for MES with local only networks that need to be balanced. To do so, load shedding or curtailment may be required, but discomfort is minimized using a new fitness metric. Furthermore, this paper also discusses how the stages of the PS algorithm align with the USEF [7] market model. The proposed solution is evaluated in Section $\mathrm{V}$ by revisiting the case from [5], but now with HPs included (shown in Fig. 1). Conclusions and directions for future work are presented in Section VI.

The main contributions of this paper are as follows:

- a scalable framework for MES optimization,

- support for load shedding and curtailment.

\section{Profile Steering Algorithm}

This section gives an overview of the PS algorithm [6] and previously published extensions to PS.

\section{A. Algorithm}

The PS algorithm [6] is a heuristic to schedule a cluster of $M$ devices for an upcoming period of $N$ time intervals. It does so by iteratively reducing the Euclidean distance between desired power profile $\vec{p}$ and aggregated power profile $\vec{x}$, i.e., minimize $\|\vec{x}-\vec{p}\|_{2}$. The zero vector is used for $\vec{p}$ to expresses that energy balance is desired.

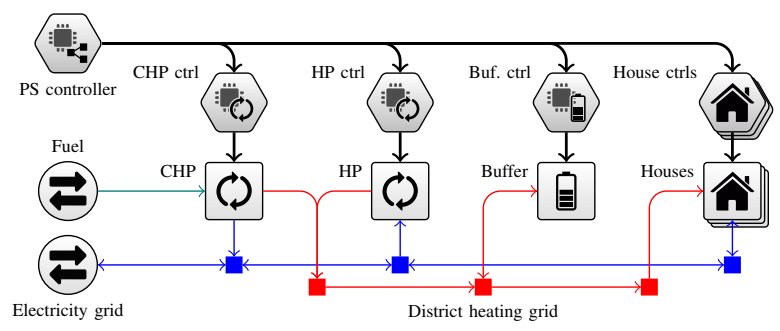

Fig. 1. DEMKit model of the MES with devices and controllers. 
Initially, each device $m \in M$ receives $\vec{p}$ and optimizes its own power profile $\vec{x}_{m}=\left[x_{m, 1}, \ldots, x_{m, N}\right]^{T}$ to minimize $\left\|\vec{x}_{m}-\vec{p}\right\|_{2}$. The PS controller receives a power profile from each connected device, resulting in $\vec{x}=\sum_{m \in M} \vec{x}_{m}$. Then, in an iterative process, the PS controller sends out a difference profile $\vec{d}=\vec{p}-\vec{x}$. Each device obtains a local desired profile $\vec{p}_{m}=\vec{d}+\vec{x}_{m}$ and optimizes its schedule to find a new optimized candidate power profile $\overrightarrow{\hat{x}}_{m}$ that minimizes $\left\|\overrightarrow{\hat{x}}_{m}-\vec{p}_{m}\right\|_{2}$. Each device calculates the improvement $e_{m}$ that is achieved by replacing $\vec{x}_{m}$ by $\overrightarrow{\hat{x}}_{m}: e_{m}=\left\|\vec{x}_{m}-\vec{p}_{m}\right\|_{2}-$ $\left\|\overrightarrow{\hat{x}}_{m}-\vec{p}_{m}\right\|_{2}$, and communicates $e_{m}$ back to the PS controller. The PS controller selects the device with the largest $e_{m}$. The corresponding device $m$ commits its own candidate profile (i.e., $\vec{x}_{m}:=\overrightarrow{\hat{x}}_{m}$ ) and PS updates $\vec{x}$ accordingly. Subsequently, a new difference profile $\vec{d}$ is obtained to repeat the process until no significant improvement is made or the maximum number of iterations is reached. The result of the algorithm is a schedule $\vec{x}_{m}$ for each device $m$. Device specific optimization algorithms are presented in [8].

\section{B. Profile Steering with Bounds}

Certain bottlenecks in the grid, referred to as congestion points, limit the possible flow of energy and therefore also the utilization of flexibility. Van der Klauw et al. [9] have extended PS to include bounds in the steering signals. Vectors $\vec{b}_{\max }$ and $\vec{b}_{\text {min }}$ indicate respectively upper and lower bounds.

After creating the initial planning, the algorithm verifies if these bounds are respected. If not, the desired profile is altered to maximize the distance to either bound, i.e. $p_{i}=\left(b_{\min , i}-\right.$ $\left.b_{\max , i}\right) / 2$ and the bounds are communicated together with the desired profile in the process. The devices use this information to create feasible schedules within their comfort constraints using their flexibility, i.e. load shedding or curtailment is not applied. Subsection III-A discusses the situation in which no feasible solution is found.

\section{Profile Steering with Multiple Commits}

Basic PS only selects one profile per iteration to ensure convergence, however, this hinders its scalability. This was evaluated in [10] and a method to reduce the computation time with many devices has been proposed. To lift this issue, only a fraction of the desired profile $\left(\frac{\vec{p}}{\mu}\right)$ is communicated to all devices, and also allowing $\mu$ candidate profiles to be accepted in each iteration. In subsequent iterations $\mu$ may be reduced, until $\mu=1$, resulting in the original PS algorithm.

\section{Profile Steering with Multiple Energy Carriers}

Schoot Uiterkamp et al. [11] extended PS by replacing the steering vectors with matrices, e.g., $\vec{x}$ becomes $\vec{X}$. Each column $\vec{X}^{c}$ represents an energy carrier $c \in C$, and the desired profiles for multiple energy carriers are sent to a device at once. The advantage of this approach is that devices, especially converters, can optimize their operation for multiple energy carriers simultaneously. In practice, the energy consumption/generation of one energy carrier depends on the consumption/generation of another energy carrier for these devices. In [11] a weight $w_{c}$ for each carrier is chosen, such that $e_{m}$ becomes a weighted sum.

\section{SUPPORTING MULTI-ENERGy SyStems}

To allow the joint optimization of multiple energy carriers, matrices have been used. However, the approach presented in [11] only minimizes the (weighted) Euclidean distance to the desired profile. This is no problem when all energy carriers have a connection to the main grid. This way, a shortage of energy can be supplied, or a surplus can be exported to the main grid. In practice, a MES often incorporates a local energy system that is not connected to a main grid. A common example is the inclusion of a district heating network. In essence, such a heat network can be considered as an islanded grid that requires balance between consumption and production at all times. This section presents the inclusion of load shedding and curtailment in PS to guarantee such balance.

\section{A. Adding Forced QoS Degradation}

Islanded networks should achieve energy balance using producers (sources), consumers (sinks), and storages (buffers). Insufficient flexibility is provided to reach balance if, after applying aforementioned bounds (Subsection II-B), the profile is still not within limits. To resolve this, a third stage is added, right after the extension presented by [9], that forces devices to perform load shedding or curtailment. We implement this by supplying an additional parameter with the steering signals: forceBalance $=$ True. However, load shedding should be limited to strictly the amount necessary to minimize the discomfort.

The comfort degradation should be spread fairly among all consumers, producers and converters. To achieve fairness, the multiple commits approach (Subsection II-C, [10]) is applied in this stage with $\mu=M$. The upper and lower bounds are also divided by $\mu$ to avoid overshoots in aggregated profile. For local only energy carriers, the bounds can be set to zero (with an $\epsilon$ margin) to indicate that there is no other grid with which energy can be exchanged.

Furthermore, device level optimization algorithms have their own objectives to maximize their comfort within the given bounds. If such comfort objectives are not in place, device optimization algorithms may (under certain conditions) propose a candidate profile that maximizes the improvement value by applying full load shedding or curtailment, which often results in minimum comfort. This is also the reason why the approach of [9] is applied first to initially avoid discomfort. Forced discomfort stage is therefore only used as a last resort to guarantee balance.

\section{B. Different Improvement Metric}

Considering the MES as a whole, the aforementioned producers and consumers may become converters. This is the case where at least two involved energy carriers are optimized using PS. Examples are HPs (converting electricity into heat), CHPs (converting gas into heat and electricity).

Converters have a special role, as they affect the profiles of multiple energy carriers simultaneously. Only implementing load shedding and curtailment is insufficient as the resulting profile, that obeys the limits for one energy carrier (e.g., to balance a heat network), may result in a negative overall improvement (e.g. a larger electricity peak with higher weight). 
Therefore, such a feasible candidate profile is never selected in the PS algorithm and hence the infeasible schedule remains.

To avoid such behaviour, a new improvement metric is introduced for the load shedding and curtailment phase of PS. Instead of the Euclidean distance from the desired profile, we use the euclidean distance from either bound. For this, we first obtain the penalty matrices $O$ and $\hat{O}$, which indicate the violation of either bound $\left(b_{\max }^{c}\right.$ and $\left.b_{\min }^{c}\right)$, for both the current profile $X_{m}$ and candidate profile $\hat{X}_{m}$ respectively. An element of the penalty for device $m$, time interval $i$, and energy carrier $c$, is defined as:

$$
o_{m, i}^{c}= \begin{cases}b_{\min , i}^{c}-x_{m, i}^{c} & \text { if } x_{m, i}^{c}<b_{\min , i}^{c} \\ x_{m, i}^{c}-b_{\max , i}^{c} & \text { if } x_{m, i}^{c}>b_{\max , i}^{c} \\ 0 & \text { otherwise. }\end{cases}
$$

The penalty elements for the candidate profile $\hat{O}$ are obtained likewise. The resulting improvement $e_{m}^{\prime}$ is obtained by calculating the improvement in Euclidean distance, similarly to the method described in [11]:

$$
e_{m}^{\prime}=\sum_{c \in C} w_{c} \cdot\left(\left\|\vec{o}_{m}^{c}\right\|_{2}-\left\|\hat{\vec{\partial}}_{m}^{c}\right\|_{2}\right)
$$

This means that, if a profile fits within bounds, its improvement is maximized and no further incentive is given to reach the desired profile (which would lead to more discomfort than strictly necessary as mentioned before). However, depending on the reaction of other devices, it may still occur that a device is able to propose a positive improvement $e_{m}$, whilst $e_{m}^{\prime} \geq 0$ (i.e. a feasible solution). Therefore, the best improvement metric $\hat{e}_{m}$ is communicated, as long as $e_{m}^{\prime} \geq 0$ :

$$
\hat{e}_{m}= \begin{cases}e_{m} & \text { if } e_{m} \geq 0 \wedge e_{m}^{\prime} \geq 0 \\ e_{m}^{\prime} & \text { otherwise }\end{cases}
$$

If no more improvement is provided, the profile is either within bounds or PS cannot find a feasible solution.

\section{Extended Heuristic}

This subsection presents an overview of the PS algorithm which includes aforementioned extension, summarized in Alg. 1 , which is based on the work presented in [6], [9]-[11]. Refer to aforementioned literature for more in depth details.

The heuristic initially starts by requesting an initial power profile (line 24), for which minimizing the Euclidean distance to the desired profile $\vec{P}$ is the objective. However, this initial plan may as well be the result of other market models. This phase can be seen as the green phase in USEF [7] (e.g. free market), where the result is the so-called A-plan, which is used for validation by distribution service operators (DSOs).

Similarly to Van der Klauw et al. [9], we check this initial profile for feasibility with given bounds (line 26). If it is not, we adapt the desired profile to maximize the distance to either bound and run the PS algorithm again with the adapted desired profile $\overrightarrow{P^{\prime}}$ (lines 27-28). This phase is similar to the yellow phase in USEF, in which congestion points are declared and the market is requested to stay within these limits. From this point on, the resulting profile is again validated with set bounds. If the initial profile is feasible (line 30), the heuristic proceeds with a regular PS as presented in [6], [11] (line 33).

However, if the planning is not feasible, the algorithm cannot find one that fits within given comfort constraints. The PS algorithm is executed one more time, but now with forceBalance $=$ True to find a feasible solution (line 31). Each device $m$ needs to adhere to local bounds $B_{\max , m}$ and $B_{\min , m}$ (see also [9]). In this stage, the adaptions to PS as presented in this Section are in place. The forced QoS aligns with the orange phase of USEF, in which the DSO is allowed to directly control devices.

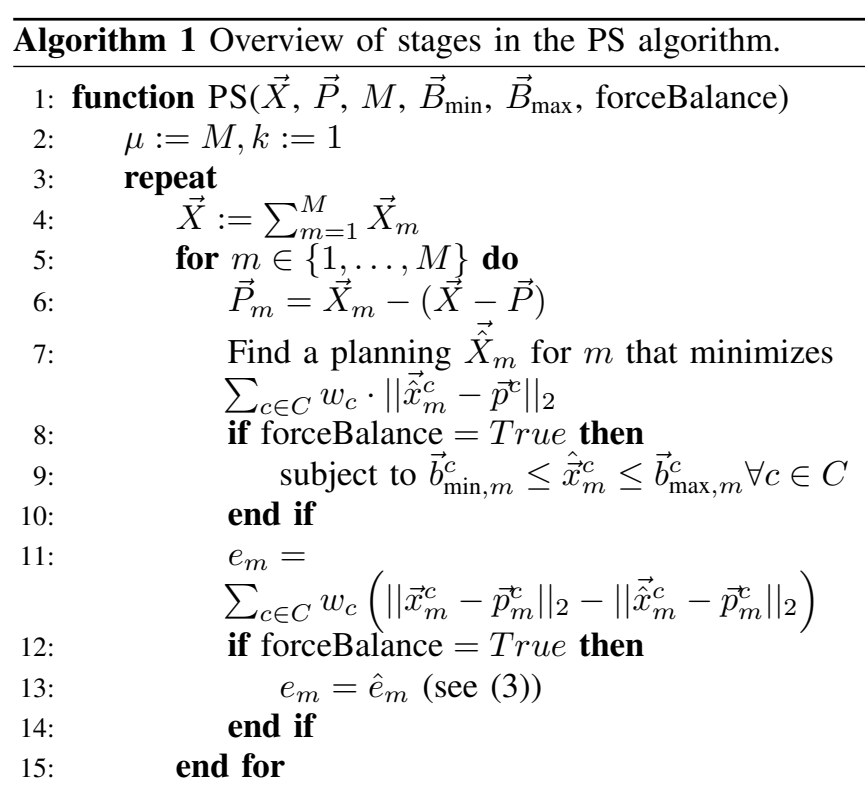

Sort devices $M$ non-increasingly based on $e_{m}$ for $m \in\{1, \ldots, \mu\}$ do $\triangleright$ Subset of devices in $M$ $\vec{X}_{m}:=\overrightarrow{\hat{X}}_{m}$ end for $\mu:=\max \left\{1,\left\lfloor\frac{\mu}{2}\right\rfloor\right\}, k:=k+1$

until $e_{1}<e_{\min } \vee k>k_{\max } \quad \triangleright$ Stopping conditions Return $\vec{X}$

end function

24: Request each device $m \in\{1, \ldots, M\}$ to minimize $\sum_{c \in C} w_{c} \cdot\left\|\vec{x}_{m}-\vec{p}^{c}\right\|_{2}$

25: $\vec{X}:=\sum_{m=1}^{M} \vec{X}_{m}$

$\triangleright$ Initial cluster consumption

26: if $\neg\left(\vec{b}_{\min }^{c} \leq \vec{x}^{c} \leq \vec{b}_{\max }^{c}\right) \forall c \in C$ then $\triangleright$ Infeasible planning 27: $\quad \vec{P}^{\prime}=\left(\frac{\vec{B}_{\min }+\vec{B}_{\max }}{2}\right) \quad \triangleright$ Adapt desired profile (see [8]) 28: $\quad \vec{X}:=\operatorname{PS}\left(\vec{X}, \vec{P}^{\prime}, M, \vec{B}_{\min }, \vec{B}_{\max }\right.$, False $)$

29: end if

30: if $\neg\left(\vec{b}_{\text {min }}^{c} \leq \vec{x}^{c} \leq \vec{b}_{\text {max }}^{c}\right) \forall c \in C$ then $\quad \triangleright$ No solution $\triangleright$ Run PS with adaptions presented in Section III 31: $\quad \vec{X}:=\operatorname{PS}\left(\vec{X}, \vec{P}^{\prime}, M, \vec{B}_{\min }, \vec{B}_{\max }\right.$, True $)$

32: else $\triangleright$ Perform standard PS (see [6], [11])

33: $\quad \vec{X}:=\operatorname{PS}\left(\vec{X}, \vec{P}, M, \vec{B}_{\min }, \vec{B}_{\max }\right.$,False $)$

end if 


\section{Converter Device Optimization}

In addition to the coordination algorithm, we also introduce a new device class, namely the converter. A buffer-converter combination was used in [5], for which the buffer optimization algorithm from [8] could be used. With separate buffers and converters, various combinations can be modeled and an optimization algorithm for converters (HPs, CHPs) is required.

In this work, we assume an ideal, linear converter with constant coefficient of performance (CoP). The optimal profile for the primary energy carrier $c^{\prime}$ (often the input energy source) can be determined as follows:

$$
x_{m, i}^{c^{\prime}}=\max \left\{b^{-}, \min \left\{\sum_{c \in C} \frac{w_{c} \cdot p_{m, i}^{c}}{y_{c}^{c^{\prime}}}, b^{+}\right\}\right\}
$$

where $y_{c}^{c^{\prime}}$ is the conversion rate from carrier $c$ to $c^{\prime}, b^{-}$ and $b^{+}$are constraining bounds, e.g., the maximum power input supported by the device or imposed bounds by the PS algorithm, the strictest bounds are applied. We only impose the PS bounds when the forceBalance option is provided in the steering signal to perform load shedding or curtailment.

\section{Evaluation}

The proposed extensions to PS are evaluated using a simulation case based on the case presented in [5]. Different variations of this use-case are tested, e.g. we vary the number of HPs and storage assets. The performance is evaluated in terms of execution time and the resulting power profile.

\section{A. Setup}

The original PS algorithm, as well as the extended version, are implemented in the DEMKit simulation software [12]. The load and generation models, as well as the device models and their flexibility are equal to the case as presented in [5]. Likewise, we simulate a full year (365 days) in 15 minute intervals. Our optimization objective is to minimize the import/export of energy, i.e., the objective desired profile matrix is filled with zeroes. The resulting electricity import export is quantified using the Euclidean distance from zero.

The difference in this test case is that we are only interested in the performance of the modified PS algorithm. To avoid further external factors, we do not use predictions. This in contrast to [5] where prediction errors, robustness and operational control are included. Hence, the results of this work are not directly comparable. Furthermore, we use ideal, loss-free

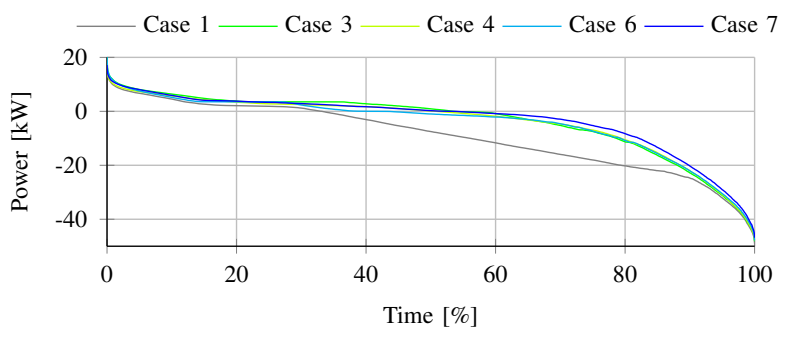

Fig. 2. Load duration curves of MES power demand without battery. devices. The simulations are performed using an Intel Core i7-5820K@4.0 GHz using a single threaded implementation.

Similar to [5], we are dealing with an islanded district heating network shared among the households. Therefore, $\vec{b}_{\text {min }}^{\text {heat }}$ and $\vec{b}$ max are restricted to a small $\epsilon$ deviation from energy balance. All devices are connected to one central PS controller. The weights for the energy carriers are: $w_{\text {electricity }}=0.5$, $w_{\text {heat }}=0.5$. One year is simulated, using time intervals of 15 minutes. A rolling horizon approach is used for the optimization, which plans the devices two days ahead (192 intervals) and performs a new planning every day (96 intervals).

\section{B. Simulation Results}

In total eight variations of the case, with different technologies, have been simulated as presented in Table I. Case 2 is similar to the work presented in [5], whereas the other cases make use of the presented PS extension by adding one or 16 HPs, a heat buffer, and battery storage. These are the flexible devices performing local optimizations in the PS coordination algorithm. Additionally, the static objects for each house (load, heat demand, and PV panels) add 48 objects to the model.

The total time of each simulation (Sim.), as well as the total time spent for optimization using PS (Opt.) are evaluated. Adding a single HP and/or buffer storage does not significantly affect the total optimization time. Moving from one to 16 individual HPs (from cases 3-5 to cases 6-8) has a significant effect, but not as pronounced as adding 16 batteries. The latter adds significant amounts of flexibility to the system, which results in additional iterations where the electricity and heat systems iteratively react to each other.

The use of 16 HPs does not change the aggregated offered flexibility, but it does change the problem presented to the PS heuristic. It results in a larger $\mu=M$, and therefore also presents a smaller fraction of the desired profile initially to the HPs. This not only results in the increase of computation time, but also consistently results in an improved objective value (Obj.) when comparing cases 3-5 with cases 6-8. This objective value is defined as $\sum w_{c} \cdot\left\|\vec{x}^{c}\right\|_{2}$.

A load duration curve of the electricity import/export of the MES is visualized in Fig. 2 and 3. These figures show that the HP can reduce the excess electricity for roughly $50 \%$ of the time, and in the best case reduces the export by $51,4 \%$. The addition of battery storage results in a nearly balanced operation for $60 \%$ of the time (Case 8), Only the last $20 \%$ shows significant overproduction, predominantly by PV, that cannot be stored in either the battery or thermal storage.

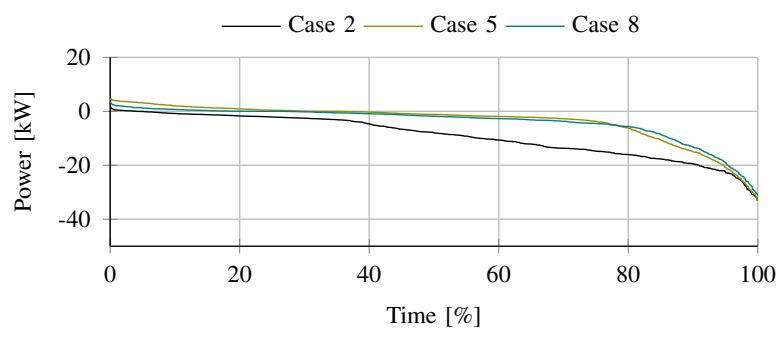

Fig. 3. Load duration curves of MES power demand with battery. 
TABLE I

NUMERICAL RESULTS OF DIFFERENT SIMULATION CASES

\begin{tabular}{|c|c|c|c|c|c|c|c|c|c|c|c|c|}
\hline \multirow[b]{2}{*}{ Case } & \multicolumn{4}{|c|}{ Technologies } & \multicolumn{3}{|c|}{ Statistics } & \multicolumn{2}{|c|}{ Energy per house } & \multicolumn{3}{|c|}{ Microgrid Energy } \\
\hline & $\begin{array}{c}\text { CHP } \\
{\left[\mathrm{kW}_{\mathrm{e} / \mathrm{th}}\right]}\end{array}$ & $\begin{array}{l}\text { Heatpump } \\
{\left[\mathrm{kW}_{\mathrm{e} / \mathrm{th}}\right]}\end{array}$ & $\begin{array}{c}\text { Buffer } \\
{\left[\mathrm{kWh}_{\mathrm{th}}\right]}\end{array}$ & $\begin{array}{l}\text { Battery } \\
{\left[\mathrm{kWh} \mathrm{h}_{\mathrm{e}}\right]}\end{array}$ & $\begin{array}{c}\text { Opt. } \\
{[\mathrm{s}]}\end{array}$ & $\begin{array}{l}\text { Sim. } \\
{[\mathrm{s}]}\end{array}$ & $\begin{array}{l}\text { Obj. } \\
\cdot 10^{6} \\
\end{array}$ & $\begin{array}{l}\text { Import } \\
{\left[\mathrm{kWh}_{\mathrm{e}}\right]}\end{array}$ & $\begin{array}{l}\text { Export } \\
{\left[\mathrm{kWh}_{\mathrm{e}}\right]}\end{array}$ & $\begin{array}{c}\mathrm{CHP} \\
{\left[\mathrm{MWh}_{\mathrm{e}}\right]}\end{array}$ & $\begin{array}{l}\text { Heatpump } \\
{\left[\mathrm{MWh}_{\mathrm{e}}\right]}\end{array}$ & $\begin{array}{c}\text { Buffer } \\
{\left[\mathrm{MWh}_{\mathrm{th}}\right]}\end{array}$ \\
\hline 1 & $30 / 60$ & - & 250 & - & 256 & 1084 & 2.86 & 654 & 5639 & 135.0 & 0.0 & 23.4 \\
\hline 2 & $30 / 60$ & - & 250 & $16 \times 4$ & 2303 & 3477 & 2.22 & 9 & 4992 & 135.0 & 0.0 & 25.9 \\
\hline 3 & $20 / 40$ & $10 / 40$ & - & - & 230 & 1047 & 2.33 & 1239 & 3256 & 71.1 & 63.9 & 0.0 \\
\hline 4 & $20 / 40$ & $10 / 40$ & 250 & - & 287 & 1123 & 2.26 & 995 & 3208 & 75.9 & 59.1 & 22.2 \\
\hline 5 & $20 / 40$ & $10 / 40$ & 250 & $16 \times 4$ & 2372 & 3564 & 1.51 & 273 & 2244 & 70.7 & 64.3 & 20.4 \\
\hline 6 & $20 / 40$ & $16 \times .6 / 2.5$ & - & - & 817 & 1835 & 2.25 & 887 & 3261 & 79.3 & 55.7 & 0.0 \\
\hline 7 & $20 / 40$ & $16 \times .6 / 2.5$ & 250 & - & 869 & 1963 & 2.12 & 1063 & 2780 & 65.3 & 69.7 & 27.7 \\
\hline 8 & $20 / 40$ & $16 \times .6 / 2.5$ & 250 & $16 \times 4$ & 3344 & 4793 & 1.39 & 92 & 2241 & 74.5 & 60.5 & 29.8 \\
\hline
\end{tabular}

\section{Discussion}

A better balance between production and consumption is achieved by exploiting the synergies between a HP and CHP. Especially the exports have significantly decreased compared to the cases without a HP. The energy passing through the buffer increases, hence the hybrid energy system uses this flexibility to act as a buffer. In most cases, the CHP produces slightly more electricity than the HP consumes, which also leads to a larger import. Although an improvement is achieved, the comparison between 1 and 16 HPs already indicates that different feasible solutions are found with more iterations as $\mu$ increases. This results in more opportunities for devices to react to each other, leading to a better solution.

However, we expect that the algorithm frequently ends up in a local minimum. The interaction between many converters and buffers is not trivial. Especially in a framework which uses device agnostic steering signals, such as PS. Visual inspection of the time series data reveal that a trivial solution with better (lower) objective value was obtainable by only changing the balance between CHP and the HP.

There are two possible directions to lift this issue, whilst keeping the strengths of device agnostic steering signals. Firstly, additional hierarchical layers can be introduced in PS, where such devices are clustered together and are seen as a single device, e.g., a buffer, to a higher level PS controller. On the other hand, the number of accepted profiles may be reduced such that it is (much) lower than $\mu$. This increases the number of iterations, but allows for more balancing interaction.

\section{CONCLUSION}

This paper presented an extension to the PS heuristic to support the optimization of MES using device agnostic steering signals, such that new devices can be integrated into the optimization framework easily. The evaluation shows that these changes result in an improved balance (objective value) and keep the optimization time limited in most cases. Furthermore, the presented method to curtail energy production is also applicable to single energy carrier systems.

However, the discussion in Subsection V-C also identifies that the results obtained are not optimal. Future work in clustering and additional parameters is advised. However, due to the dependencies between energy carriers, this may not be trivial. Future work should investigate whether a practical method or algorithm can be applied to automate such clustering and the selection of parameters values, which result in (near) optimal results within reasonable computation time.

\section{ACKNOWLEDGMENT}

This work is supported by Dutch national program TKI iDeego (project ORTEP) and the European Fund for Regional Development (Project OP-Oost PROJ-00939).

\section{REFERENCES}

[1] P. Mancarella, "MES (multi-energy systems): An overview of concepts and evaluation models," Energy, vol. 65, no. C, pp. 1-17, 2014

[2] E. A. Martínez Ceseña and P. Mancarella, "Energy systems integration in smart districts: Robust optimisation of multi-energy flows in integrated electricity, heat and gas networks," IEEE Transactions on Smart Grid, vol. 10, no. 1, pp. 1122-1131, Jan 2019.

[3] E. A. Martínez Ceseña, C. Heltorp, and P. Mancarella, "A transactive energy modelling and assessment framework for demand response business cases in smart distributed multi-energy systems," Energy, 2018.

[4] P. Booij, V. Kamphuis, O. van Pruissen, and C. Warmer, "Multi-agent control for integrated heat and electricity management in residential districts," in 4th International Workshop on Agent Technologies for Energy Systems (ATES), a workshop of the 12th International Conference on Autonomous Agents and Multiagent Systems (AAMAS), Minnesota, 2013.

[5] B. Homan, G. Hoogsteen, S. Nebiolo, J. L. Hurink, and G. J. Smit, "Maximizing the degree of autarky of a 16 house neighbourhood by locally produced energy and smart control," Sustainable Energy, Grids and Networks, vol. 20, 2019

[6] M. E. T. Gerards, H. A. Toersche, G. Hoogsteen, T. van der Klauw, J. L. Hurink, and G. J. M. Smit, "Demand side management using profile steering," in PowerTech, 2015 IEEE Eindhoven, June 2015, pp. 1-6.

[7] Universal Smart Energy Framework, "USEF: The framework explained," [Online] Available: https://www.usef.energy, last accessed on 05-022020.

[8] T. van der Klauw, "Decentralized energy management with profile steering - resource allocation problems in energy management," $\mathrm{Ph} . \mathrm{D}$. dissertation, University of Twente, Enschede, The Netherlands, May 2017, CTIT Ph.D. thesis Series No. 17-424.

[9] T. van der Klauw, M. E. T. Gerards, G. Hoogsteen, G. J. M. Smit, and J. L. Hurink, "Considering grid limitations in profile steering," in 2016 IEEE International Energy Conference (ENERGYCON), Leuven, April 2016.

[10] G. Hoogsteen, M. E. T. Gerards, and J. L. Hurink, "On the scalability of decentralized energy management using profile steering," in 2018 IEEE PES Innovative Smart Grid Technologies Conference Europe (ISGTEurope), Oct 2018.

[11] M. H. H. Schoot Uiterkamp, G. Hoogsteen, M. E. T. Gerards, J. L. Hurink, and G. J. M. Smit, "Multi-commodity support in profile steering," in 2017 IEEE PES Innovative Smart Grid Technologies Conference Europe (ISGT-Europe), Sep. 2017.

[12] G. Hoogsteen, J. L. Hurink, and G. J. M. Smit, "DEMKit: a decentralized energy management simulation and demonstration toolkit," in 2019 IEEE PES Innovative Smart Grid Technologies Europe (ISGT-Europe), Sep. 2019. 